Nataša Radusin Bardić*

Faculté de Philosophie et Lettres

Université de Novi Sad
UDK : 811.133.1'34:371.3

DOI: $10.19090 /$ gff.2021.3.155-174

originalni naučni rad

\title{
SUR LA MISE EN CONTEXTE DES ACTIVITÉS DE PHONÉTIQUE DANS LES MÉTHODES DE FLE S'INSCRIVANT DANS UNE PERSPECTIVE ACTIONNELLE
}

Notre recherche portera sur l'analyse de la mise en contexte des activités de phonétique dans cinq méthodes de français langue étrangère (FLE) publiées entre 2011 et 2018 qui s'inscrivent dans la lignée des approches communicative et actionnelle et s'appuient sur les principes pédagogiques décrits par le CECR de 2001 (Nouveau Rond-Point 1, Écho A1, Saison 1, Cosmopolite 1 et Défi 1). L'objectif de cette recherche sera double. D'une part, nous voulons savoir quelle place est accordée aux activités de phonétique dans chacune des cinq méthodes sélectionnées. D'autre part, nous voulons déterminer à quel point les activités de phonétique y sont mises en contexte vu que dans le CECR une large attention est consacrée au rôle du contexte dans toute forme d'usage et d'apprentissage d'une langue étrangère. Afin de pouvoir mieux comparer les résultats obtenus et mieux comprendre l'importance donnée à la contextualisation des activités de phonétique dans les méthodes de FLE choisies, notre analyse sera basée sur des critères qualitatifs aussi bien que quantitatifs.

Mots clés : français langue étrangère, phonétique française, activités de phonétique, mise en contexte, approche communicative, perspective actionnelle.

\section{INTRODUCTION}

Dans les années 1960-1970, à l'époque où dominait la méthodologie structuro-globale audio-visuelle (SGAV), connue pour sa méthode originale de correction phonétique (la méthode verbo-tonale), la phonétique a été considérée comme l'un des piliers fondamentaux de l'enseignement/apprentissage d'une langue étrangère. Vers la fin des années 1970, avec l'avènement de l'approche communicative, la phonétique commence à perdre son rôle prépondérant. De nombreux spécialistes du français langue étrangère (FLE), didacticiens et phonéticiens, ont essayé d'éclaircir les raisons de sa marginalisation en rapport avec l'apparition de

\footnotetext{
*natasa.radusin.bardic@ff.uns.ac.rs
} 
l'approche communicative (Guimbretière, 1994 : 48-49 ; Champagne-Muzar \& Bourdages, 1998 : 17 ; Lauret, 2007 : 35). En favorisant la spontanéité des échanges oraux, l'approche communicative a inévitablement entraîné un changement de critère suivant : " le critère de la performance optimale (identité avec le système-cible) a été remplacé par le critère d'acceptabilité maximale des performances pourvu qu'elles n'entravent pas la communication »(Gallazi-Matasci \& Pedoya, 1983 : 40). L'enseignement de la prononciation minutieux et systématique, tel qu'il avait été à l'époque de la méthodologie SGAV, n'a pas su «prendre ce virage pédagogique » (Porcher, 1987 : 136), adapter ses recherches (Gallazi-Matasci \& Pedoya, 1983 : 39) et trouver « une voie méthodologique propre et conforme aux nouvelles options de la didactique des langues » (Callamand \& Pedoya, 1984 : 56). Ce n'est qu'à partir des années 1990 qu'on assiste à " un retour en force de la correction phonétique en classe de langue » (Abry \& Veleman-Abry, 2007 : 7). Aujourd'hui « aucune méthode récente ne fait plus l'impasse sur la prononciation » (Cuq \& Gruca, 2008 : 179) bien que la phonétique continue à être « trop souvent jugée ennuyeuse par les apprenants et considérée comme une science rébarbative par les enseignants » (Cuq \& Gruca, 2008 : 179). À la différence des années 1960-1970 où l'usage de la méthode verbo-tonale de correction phonétique (dont « un des principes moteurs [...] consiste [...] à éduquer l'audition et à faire reproduire un modèle par l'imitation et la répétition ») excluait la méthode articulatoire (reposant "sur une connaissance approfondie du fonctionnement de l'appareil phonatoire ») (Cuq \& Gruca, 2008 : 180), les pratiques actuelles tendent plutôt vers l'éclectisme en combinant ces deux approches selon les besoins des apprenants (Cuq \& Gruca, 2008 : 181, Billières, 2014, para. 1). Même s'il est rare « que les enseignants prennent le temps de développer à ce point une séquence phonétique », l’ordre privilégié des pratiques phonétiques en classe de FLE est en général celui où la perception précède la production et l'exposition à l'oral précède l'exposition au code graphique (Lauret, 2007 : 133).

Avant d'exposer les objectifs de notre recherche portant sur l'analyse de la mise en contexte des activités de phonétique dans les méthodes de FLE s'inscrivant dans la perspective actionnelle, nous devons expliquer notre choix terminologique concernant «les activités de phonétique ». En didactique du FLE, le terme d'exercice est " souvent employé au même sens qu'activité d'apprentissage » (Cuq, 2008 : 94). Cependant, le Cadre européen commun de référence pour les langues : apprendre, enseigner, évaluer (CECR, 2001) 
distingue bien clairement le terme d'exercice du terme d'activité en associant le premier (qualifié de «formel ») « aux exercices hors contexte $»^{1}$ et le second « aux exercices en contexte» (Robert, 2008 : 86). Il faut souligner le fait que dans le CECR une large attention est consacrée au rôle du contexte dans toute forme d'usage et d'apprentissage d'une langue. Le contexte y est défini comme « la multitude des événements et des paramètres de la situation (physiques et autres), propres à la personne mais aussi extérieurs à elle, dans laquelle s'inscrivent les actes de communication » (CECR, 2001 : 15). Dans les pratiques phonétiques en classe de FLE, Bertrand Lauret propose une typologie selon laquelle les exercices de prononciation « exercent en perception et production par la réitération de l'action suivant un modèle » à la différence des activités de prononciation qui «n'ont généralement pas de caractère répétitif, ni de caractère évaluatif » et qui « incitent l'apprenant à la réflexion et à la création » (Lauret, 2007 : 138). Malgré ces efforts de clarification terminologique, on constate qu'en général « dans les méthodes actuelles, le terme d'exercice est tantôt associé, tantôt opposé à celui d'activité » (Robert, 2008 : 86), y compris dans les supports phonétiques. À titre d'exemple, dans la méthode Écho $A 1$ « des exercices de prononciation » (ÉC1, 2013 : IV) et " des activités de prononciation » (ÉC1-GP, 2013 : IV) sont utilisés comme des synonymes. Par contre, dans la méthode Défi 1 on trouve uniquement « des exercices » de phonétique dans le Cahier d'exercices (DF1-GP, 2018, DF1-CA, 2018) tandis que dans la méthode Cosmopolite 1 on trouve uniquement " des activités de phonétique " tout aussi bien dans le Livre de l'élève que dans le Cahier d'activités (COS1-GP, 2017 : 3). Comme dans la plupart des méthodes analysées nous avons noté l'usage prépondérant du terme " des activités de phonétique » (NRP1-CA, 2011 ; ÉC1-GP, 2013 ; SN1-GP, 2014 ; COS1, 2017), nous l'avons aussi adopté dans notre recherche tout en sachant qu'il n'est pas toujours conforme à la définition des termes d'exercice et d'activité donnée dans le CECR (2001).

${ }^{1}$ Dans le Guide pour les utilisateurs du CECR, des « exercices spécifiquement axés sur la manipulation décontextualisée des formes » sont regroupés sous le nom des "tâches de pré-communication pédagogique » (Trim, 2001 : 153). Jean-Pierre Robert, Évelyne Rosen et Claus Reinhardt adoptent cette appellation, mais contrairement au Guide pour les utilisateurs du CECR, ils estiment que «les tâches de pré-communication pédagogique » peuvent être contextualisées ou non (Robert-Rosen \& Reinhardt, 2011 : 138). 
En partant du constat de Jean-Pierre Cuq et Isabelle Gruca que « la phonétique ne constitue plus un moment de la classe : elle est intégrée à chaque phase et est contextualisée » (Cuq \& Gruca, 2008 : 181), l'objectif de notre recherche est double. D'une part, nous voulons évaluer la place qui est généralement accordée aux activités de phonétique (portant tout aussi bien sur l'aspect segmental que sur l'aspect suprasegmental du discours) dans les méthodes de FLE qui s'appuient sur les recommandations du CECR (2001). D'autre part, nous voulons savoir à quel point les activités de phonétique y sont réellement mises en contexte, c'est-à-dire proposées «à l'intérieur d'une situation de communication ou d'un texte » (Bertocchini \& Costanzo, 2008 : $192)^{2}$.

\section{CORPUS ET MÉTHODOLOGIE DE LA RECHERCHE}

Notre corpus se compose de cinq méthodes de FLE destinées aux apprenants adultes ou grands adolescents du niveau débutant (A1) publiées entre 2011 et 2018 par quatre maisons d'édition françaises spécialisées en FLE (CLE International, Didier, Hachette, Maison des langues) : Nouveau Rond-Point 1 (NRP1, 2011), Écho A1 (ÉC1, 2013), Saison 1 (SN1, 2014), Cosmopolite 1 (COS1, 2017) et Défi 1 (DF1, 2018). Toutes ces méthodes s'inscrivent dans la lignée des approches communicative et actionnelle et s'appuient sur les principes pédagogiques décrits par le CECR de 20013. Les mêmes critères de sélection des méthodes (niveau de langue, public, outil de référence) devraient nous permettre de mieux suivre et comparer les principes de base de la mise en contexte des activités de phonétique dans les méthodes choisies.

Dans notre recherche, nous avons analysé les activités de phonétique dans l'ensemble pédagogique qui était pour chaque méthode composé d'un livre de l'élève, d'un cahier d'activités et d'un guide pédagogique. Nous avons prêté une attention particulière à l'organisation générale des activités de

2 D'après Paola Bertocchini et Edvige Costanzo, l'enseignement de la grammaire est contextualisé si « la règle est proposée à l'intérieur d'une situation de communication ou d'un texte » ou décontextualisé si «la règle est étudiée pour elle-même, hors contexte » (Bertocchini \& Costanzo, 2008 : 192).

${ }^{3}$ En parlant du CECR, il faut aussi mentionner que dans son volume complémentaire de 2018 nous trouvons un nouveau descripteur de la maîtrise du système phonologique (Conseil de l'Europe, 2018 : 142) en remplacement de celui de 2001 (CECR, 2001 : 92). 
phonétique, à l'importance accordée à la mise en contexte des activités de phonétique (explicitée parfois dans les avant-propos des méthodes) ainsi qu'à l'examen des différentes modalités de leur contextualisation. Suite à notre analyse qualitative, nous avons aussi mené une étude statistique pour mieux comprendre le rapport entre les activités de phonétique en contexte ou hors contexte dans chacune des méthodes de FLE analysées, ainsi que pour pouvoir mieux comparer les résultats obtenus.

\section{ANALYSE DE LA MISE EN CONTEXTE DES ACTIVITÉS DE PHONÉTIQUE DANS LES MÉTHODES DE FLE SÉLECTIONNÉES (2011-2018)}

Avant de donner les résultats statistiques de l'analyse de notre corpus, nous voudrions présenter brièvement chaque méthode séparément en ordre croissant selon l'importance qu'elles donnent aux activités de phonétique et à leur mise en contexte.

\section{Nouveau Rond-Point 1}

Le Nouveau Rond-Point (NRP1, 2011) suit la tradition de sa précédente édition (Rond-Point, 2004) connue comme la première méthode à avoir introduit la perspective actionnelle dans la didactique du FLE. Dans l'Avantpropos du Livre de l'élève du NRP1, en parlant des ressources linguistiques au service de la communication et de la réalisation de la tâche, les auteurs ne mentionnent que des ressources lexicales et grammaticales (NRP1, $2011: 3$ ). Il est intéressant de noter que nous ne trouvons aucune activité de phonétique dans le Livre de l'élève et, par conséquent, presque aucune mention de la compétence phonétique et phonologique dans le Guide pédagogique (excepté dans les Grilles d'évaluation du DELF A1 et A2). Pour compenser ce manque, les auteurs de NRP1 proposent des activités de "Phonétique et orthographe » pour chaque unité dans le Cahier d'activités (au total il y a 25 activités dans cette rubrique). Quant au contenu des activités de phonétique, elles portent plus sur le niveau segmental (voyelles et consonnes, sans semi-voyelles) que suprasegmental (intonation, enchaînements consonantique et vocalique). Dans ce premier groupe, contrairement à nos attentes, il y a plus d'activités consacrées aux consonnes $\left(\mathrm{s} / \mathrm{z}, \int / 3, \mathrm{~s} / \int\right.$ ) et aux groupes consonantiques (tR/gR/kR, bl/pl) qu'aux voyelles (voyelles nasales: $\tilde{\mathrm{a}} / \tilde{\mathrm{o}} / \tilde{\varepsilon} / \tilde{\mathrm{e}}$ et voyelles fermées : i/y/u). Disons aussi que, bien qu'on insiste sur la contextualisation 
tout au long de la méthode, les auteurs ne semblent pas avoir pris soin d'appliquer ce même principe aux activités de phonétique ; en effet, on n'en trouve que 3 sur 25 (12\%) mises en contexte dans le Cahier d'activités. Voici un des nombreux exemples d'activités de phonétique hors contexte (1a) et un des rares exemples d'activité de phonétique en contexte (1b) (ce dernier portant sur la relation phonie-graphie) que nous trouvons dans le Cahier d'activités de la méthode NRP1 :

Exemple 1 : Activité de phonétique hors contexte (NRP1-CA, $2011: 82$ )

\section{LE $[\mathbf{R}]$}

Écoutez les exemples suivants puis répétez-les.

1. La rue est barrée après le croisement.

2. Il travaille trop.

3. Ce riz est très bon.

4. Ce camion est mal garé.

5. Je crois que Ronan est à Rennes.

6. Sophie vient de Namur.

7. Ils se sont vus au bar.

8. Elle est caissière.

9. Elle adore dormir.

10. C'est une bonne performance artistique.
Exemple 2: Activité de phonétique en contexte (NRP1-CA, $2011:$ 74)

\section{LA CUISINE FRANÇAISE, ÇA N'EXISTE PAS !}

Complétez les mots du texte et indiquez les liaisons qui provoquent le son [z].

Depuis des années, on nous explique que la cui....ine fran....ai....e est unique au monde, mais....i vous êtes déjà allé en Fran....e, vous avez ....ertainement remarqué que la ga....tronomie n'était pas au menu tous les jours.

Dans les familles, on mange des plats très variés même....i le poulet-frites ....ést in...tallé dans les habitudes de nombreu....es familles.....es plats changent en fonction de la région. Et puis, beaucoup ont adopté des re....ettes venues de l'immigration comme le cou....coul..., la paella ou les chiches-kebabs.

\section{Cosmopolite 1}

La méthode Cosmopolite 1 (COS1, 2017) est organisée en 52 leçons (réparties en 9 dossiers, y compris le Dossier 0) pour lesquelles on définit les types et les genres de discours, les savoir-faire et les savoir-agir, ainsi que les ressources linguistiques. Nous remarquons que les éléments grammaticaux et lexicaux sont précisés pour la quasi totalité des leçons tandis que les éléments phonétiques ne le sont que pour 33 leçons. Les activités de phonétique, aussi bien celles regroupées dans la rubrique « Sons du français » que celles qui se trouvent en annexe dans le "Précis de phonétique - phonie-graphie ", portent plus sur les éléments segmentaux (surtout sur les voyelles, mais aussi sur les consonnes et groupes consonantiques sans oublier les semi-voyelles) que suprasegmentaux (accentuation, rythme, intonation, liaison). Un certain nombre d'activités est aussi consacré aux aspects morphophonologiques du discours (marques orales du singulier/pluriel, différenciation présent/passé). Dans le Guide pédagogique on explique qu'un travail sur les sons du français 
est intégré aux leçons et que les différents aspects (prosodie, continuité, phonèmes, rapport phonie-graphie) s'appuient sur les corpus dégagés dans les supports et apparaissent de manière progressive et contextualisée (COS1-GP, 2017 : 6). Suite à une analyse de l'organisation des activités de phonétique dans la méthode COS1, nous remarquons qu'une large majorité de ces activités est cependant décontextualisée (70\% hors contexte contre $30 \%$ en contexte dans le Livre de l'élève, 91\% hors contexte contre 9\% en contexte dans le Cahier d'activités) bien qu'elles soient composées des éléments tirés d'autres activités organisées d'une manière contextualisée et cohérente.

Voici un exemple d'une activité de phonétique hors contexte. Suite à une activité de discrimination sonore des consonnes [s] / [z] (7a), on demande aux apprenants de faire quatre phrases avec «ils sont», " elles sont», « ils ont», « elles ont » sans aucune autre précision que formelle (7b):

Exemple 3 : Activité de phonétique hors contexte (COS1-CA, 2017 : 13)

\section{Sons du français Le son $[z]$ et la liaison verbale avec nous, vous, lls, elles}

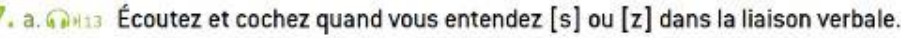

Exemple : Elles sont en classe.

\begin{tabular}{|c|c|c|c|c|c|c|c|}
\hline Phrases & Exemple & 1 & 2 & 3 & 4 & 5 & 6 \\
\hline$[\mathrm{s}]$ & $x$ & & & & & & \\
\hline$[\mathrm{z}]$ & & & & & & & \\
\hline
\end{tabular}

b. Par deux. Préparez quatre phrases avec «ils sont », « elles sont », «ils ont » et « elles ont ». Lisez les phrases, votre camarade complète le tableau.

Nous présentons aussi ci-dessous deux rares activités de phonétique, proprement dit contextualisées (en relation avec d'autres activités ellesmêmes organisées d'une manière contextualisée et cohérente) : 
Exemple 4a : Activité de phonétique en contexte (COS1, $2017:$ 77)

9. 5 or

L'intonation pour exprimer plusieurs actions

a. Âr 85 Écoutez à nouveau les moments de la journée de Natasha. Natasha parle en continu, faites un geste de la main vers le haut $\bar{~}$. Natasha marque une pause, faites un geste de la main vers le bas $x$. Exemple: Je me réveille à six heures. \}

b. An 86 Écoutez. Complétez comme dans l'exemple avec $\longrightarrow$ et et répétez.

Exemple : Le matin, Natasha se lève, $\sim$ regarde le ciel, fait du sport et se douche.<smiles>C=IC</smiles>

1. Le matin, Marc se lève, il se douche, il se rase et il s'habille.

2. Le soir, Natasha regarde une série, elle lit, elle écrit, elle se couche et elle s'endort.
Exemple 4b : Activité de phonétique en contexte (COS1, 2017 : 96)

Identifier le e muet

Recopiez ces extraits [doc. 1]. Soulignez la lettre $e$ quand elle n'est pas prononcée.

Exemple: Ils ont passé un reportage sur Ahmadou Kourouma.

1. Le reportage raconte sa vie.

2. II a écrit cinq livres pour adultes, tous des succès, et aussi des livres pour la jeunesse.

3. Mais son dernier livre, Quand on refuse on dit non, est sorti après sa mort, en 2004.

Écoutez les extraits. Répétez.

Écho $A 1$

Dans la méthode Écho $A 1$ (ÉC1, 2013), parmi les ressources linguistiques qui sont subordonnées aux savoir-faire à acquérir, en plus des éléments grammaticaux et lexicaux, nous trouvons aussi des éléments phonétiques. Ces derniers figurent dans le Livre de l'élève dans deux rubriques présentes dans chacune des douze leçons réparties en trois unités : «À l'écoute de la grammaire» (faisant partie des pages Ressources) et "Sons, rythmes, intonations» (faisant partie des pages Simulations). Comme le nom de la première rubrique l'indique («À l'écoute de la grammaire»), une attention particulière a été prêtée aux aspects morphophonologiques du discours (marques orales du masculin/féminin et du singulier/pluriel, différenciation présent/passé). 
Après une analyse du contenu de toutes les activités de phonétique du Livre de l'élève, nous constatons qu'ils portent de façon équilibrée sur les éléments segmentaux (aussi bien vocaliques que consonantiques) et suprasegmentaux (rythme, intonation, enchaînement, liaison). Il est important de noter que les auteurs de la méthode ÉC1 ont veillé à mettre la majorité des activités de phonétique du Livre de l'élève en contexte (81\%). Nous ne donnerons ici que les deux exemples suivants :

Exemple 5a : Activité de phonétique en contexte (ÉC1, $2013: 115)$

\section{(1) A l'écoute de la grammaire}

0 Son [y] et rythme de la négation « ne ... plus »

Accro aux jeux vidéo

Il ne sort plus de son studio

Il ne lit plus. C'est inutile

Il ne dort plus. Il ne mange plus

C'est ridicule
Exemple $5 \mathrm{~b}$ : Activité de phonétique en contexte (ÉC1, 2013 : 115)

\section{(1) Sons, rythmes, intonations}

Différenciez et prononcez [ø] et [\%].

Recette pour un roman policier

Dans un immeuble de banlieue

Installez un vieux professeur,

Un docteur mystérieux

Qui vit avec son neveu et sa sœur,

Une chanteuse aux cheveux bleus

Amoureuse d'un jeune acteur

À neuf heures vingt-deux

Organisez un meurtre

Et faites entrer un inspecteur

Curieux et courageux

En plus des activités de phonétique du Livre de l'élève, les apprenants ont à leur disposition celles du Cahier personnel d'apprentissage mais il faut dire que ces dernières sont moins mises en contexte que les premières $(80 \%$ hors contexte contre $20 \%$ en contexte).

\section{Saison 1}

La méthode Saison 1 (SN1, 2014) couvre le niveau A1 (unités 0 à 6) et le début du niveau A2 (unités 7 à 9). Parmi " les singularités de la méthode », les auteurs citent « la très belle place accordée » au lexique et à la grammaire, mais aussi à la phonétique qui est «travaillée régulièrement et en contexte » (SN1, 2014: 4). Dans le Livre de l'élève, il y a 62 activités de phonétique (sans compter les activités d'observation des schémas articulatoires) qui portent plus sur les éléments segmentaux (surtout sur les voyelles, mais aussi sur les consonnes) que suprasegmentaux (accentuation, syllabation, groupes 
rythmiques, enchaînements vocalique et consonantique, intonation). Un certain nombre d'activités est aussi consacré aux aspects morphophonologiques du discours (marques orales du singulier/pluriel). Dans 8 sur 9 unités (y compris l'unité 0), la phonétique est travaillée en 3 étapes : la conceptualisation (dans la rubrique Tendez l'oreille l'apprenant est invité à repérer un son en contexte et à l'oral, voir l'exemple 6), l'appropriation (des activités d'écoute, de discrimination et de répétition des sons découverts en contexte) et la production (dans le cadre des Ateliers d'expression orale, il y a une partie de préparation à la production orale réservée aux activités phonétiques : un échauffement phonétique avec un virelangue et une activité de prononciation en contexte) $)^{4}$.

Exemple 6 : Activité de phonétique en contexte (SN1, 2014:96)

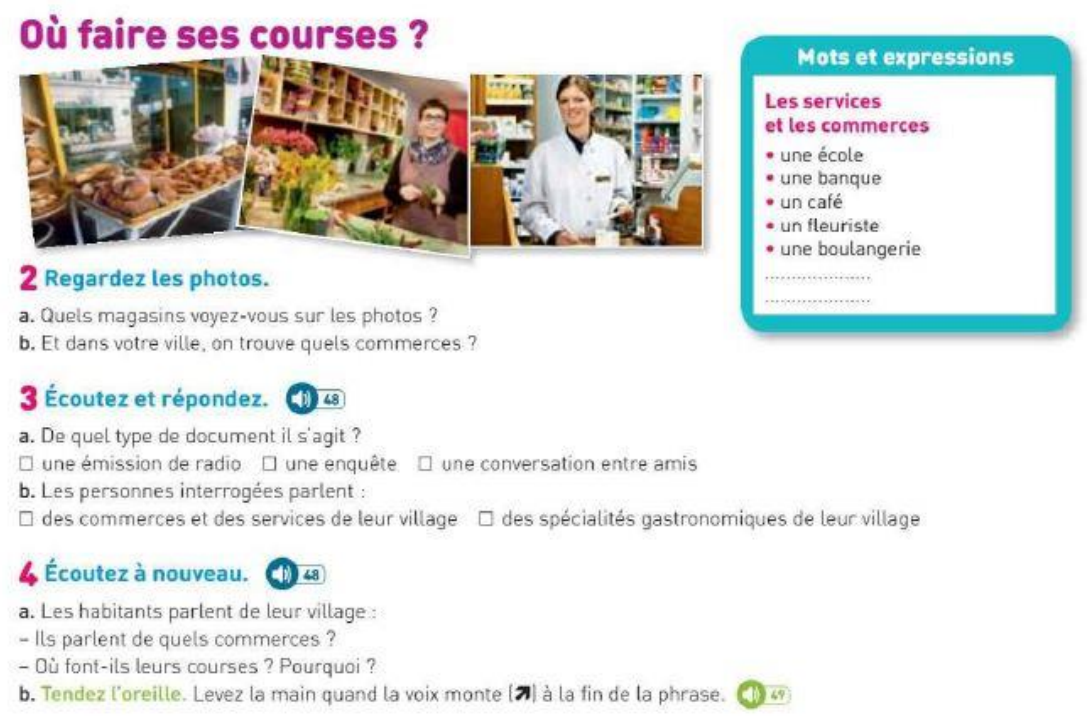

Il faut noter que grâce à la rubrique Tendez l'oreille (au nombre de deux par unité) l'apprenant est exposé aux sons en contexte sans recours à l'écrit ce

\footnotetext{
4 Dans la méthode Édito $A 1$ (ÉD1, 2016) la phonétique est aussi intégrée dans chaque unité et propose une démarche contextualisée et progressive (ÉD1, 2016:3, 5) répartie en trois étapes : l'échauffement, le fonctionnement et l'entraînement (ÉD1-GP, 2016 : 6). Cependant il faut noter que la dernière étape n'est pas subordonnée à une activité d'expression orale comme c'est le cas dans la méthode Saison 1.
} 
qui lui permet de «développer des stratégies d'écoute efficaces » (SN1-GP, 2014 : 4). En plus des trois étapes du travail sur la phonétique citées ci-dessus, on pourrait même ajouter qu'il y en a une quatrième : la «consultation de la référence » (SN1-GP, 2014 : 10). L'enseignant a à sa disposition tout au long de la méthode (dans la rubrique Point étape: Phonétique) les schémas articulatoires et des symboles simplifiés remplaçant un métalangage complexe (par exemple, on se sert d'une flèche tournée vers la gauche « $\leftarrow$ » pour marquer un son antérieur et d'une flèche tournée vers la droite « $\rightarrow$ » pour marquer un son postérieur). Si nous y ajoutons le Précis de phonétique qui se trouve à la fin du Livre de l'élève (SN1, 2014: 188-190), les activités de phonétique dans le Cahier d'activités et les outils complémentaires en ligne (le service Labo de langue, les applications nomades Oral et Dico), nous comprenons pourquoi les auteurs de la méthode Saison 1 disent que l'enseignant dispose d'« un kit d'outils » grâce auquel il peut «aborder la phonétique en classe sans complexe $»^{5}$ (SN1-GP, 2014 : 10-11).

Suite à une analyse de la mise en contexte des activités de phonétique, nous constatons qu'il y a un équilibre entre les activités contextualisées (48\%, à savoir les activités portant sur la découverte des sons en contexte et sur leur production dans le cadre des activités d'expression orale) et celles qui sont décontextualisées (52\%, à savoir les activités portant sur la discrimination et la répétition des sons découverts en contexte pour faciliter leur appropriation). Dans le Guide pédagogique, les auteurs expliquent pourquoi il est important de traiter la phonétique systématiquement en contexte : c'est parce que de cette manière "chaque point travaillé prend appui sur les situations de communication et les outils linguistiques de l'unité, ce qui permet d'ancrer l'ensemble des apprentissages en en facilitant la mémorisation " (SN1-GP, 2014 : 10). Dans le Cahier d'activités, nous trouvons un nombre plus important des activités de phonétique qui sont décontextualisées (78\% hors contexte contre $22 \%$ en contexte) parmi lesquelles figurent aussi les activités ludiques qui sont en général censées stimuler la motivation chez les apprenants. Nous donnons ci-dessous un exemple d'une activité ludique qui se trouve à la dernière unité du Livre de l'élève (SN1, 2014 : 176). Il s'agit d'une activité

${ }^{5}$ À noter que ce commentaire pourrait sous-entendre le fait que l'enseignement de la phonétique en classe de FLE nécessite une bonne préparation qui permette à l'enseignant d'aborder plus librement la phonétique en classe. 
appelée «Le jeu des sons » qui est une sorte de bilan phonétique et «qui permet de réutiliser l'ensemble des connaissances acquises lors des points phonétiques de chaque unité » (SN1-GP, 2014 : 261) :

Exemple 7 : Activité de phonétique ludique (SN1, $2014:$ 176)

\section{Phonétique}

> Le jeu des sons • Lancez les dés et jouez. Le premier arrivé a gagné !

\begin{tabular}{|c|c|c|c|c|c|}
\hline $\begin{array}{c}13 \\
\text { Avancez } \\
\text { de } 2 \text { cases. }\end{array}$ & $\begin{array}{c}12 \\
\text { Trouvez un mot } \\
\text { avec le son [ई]]. }\end{array}$ & $\begin{array}{c}119 \\
\text { Prononcez! } \\
\text { Ulysse imite une } \\
\text { minute Alice. }\end{array}$ & $\begin{array}{c}10 \\
\text { Reculez } \Rightarrow \\
\text { de } 3 \text { cases. }\end{array}$ & $\begin{array}{l}9(0) 84 \\
\text { Choisissez ! } \\
\square \text { ton plat } \\
\square \text { ton plan }\end{array}$ & $\begin{array}{l}8(4) 84 \\
\text { Choisissez ! } \\
\square \text { un jus } \\
\square \text { un jeu }\end{array}$ \\
\hline $\begin{array}{l}148 \\
\text { Prononcez! } \\
\text { Il fit faire vingt } \\
\text { verres de vin fins }\end{array}$ & ARRIVIEE & $\begin{array}{c}22 \\
\text { Je passe } \\
\text { mon tour. }\end{array}$ & $\begin{array}{l}21(0) 86 \\
\text { Choisissez! } \\
\square \text { la lame } \\
\square \text { la rame }\end{array}$ & 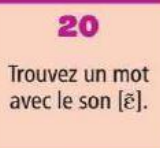 & $\begin{array}{c}7 \\
\text { Passez } \\
\text { votre tour. }\end{array}$ \\
\hline $\begin{array}{c}15 \\
\text { Passez } \\
\text { votre tour. }\end{array}$ & $\begin{array}{c}16 \\
\text { Trouvez un mot } \\
\text { avec le son [ã]. }\end{array}$ & $\begin{array}{l}17 \\
\text { Choisissez! } \\
\square \text { cent bains } \\
\square \text { cent bancs }\end{array}$ & $\begin{array}{c}18 \\
\text { Prononcez! } \\
\text { Ursule l'ours russe } \\
\text { assure! }\end{array}$ & $\begin{array}{c}19 \\
<\text { Reculez } \\
\text { de } 4 \text { cases. }\end{array}$ & $\begin{array}{c}6 \otimes \\
\text { Prononcez! } \\
\text { Le sage change en } \\
\text { s'assagissant. }\end{array}$ \\
\hline DEpART & $\begin{array}{l}1 \\
\text { Choisissez ! } \\
\square \text { un élève } \\
\square \text { une élève }\end{array}$ & $\begin{array}{c}2 \\
\text { Prononcez! } \\
\text { Le ton de tonton } \\
\text { monte. }\end{array}$ & \begin{tabular}{l}
\multicolumn{1}{c}{$\mathbf{3}$} \\
Avancez $\Rightarrow$ \\
de 2 cases.
\end{tabular} & $\begin{array}{l}4(0) 84 \\
\text { Choisissez! } \\
\square \text { le théâtre } \\
\square \text { les théâtres }\end{array}$ & $\begin{array}{l}\mathbf{5} \\
\text { Trouvez un mot } \\
\text { avec le son [y]. }\end{array}$ \\
\hline
\end{tabular}

Défi 1

Dans l'avant-propos de la méthode Défi 1 (DF1, 2018), les auteures expliquent qu'un défi particulier lors de l'élaboration de ladite méthode consistait « à proposer des documents intéressants où la langue est utilisée en contexte, tout en étant abordable pour des apprenants de niveau A1 » (DF1, 2018 : 2). Voulant mettre à profit les habitudes numériques des apprenants, les auteures ont mis en ligne ${ }^{6}$ un environnement numérique complet de la méthode ainsi qu'un grand nombre de ressources complémentaires (« des défis supplémentaires ») parmi lesquelles notamment des capsules vidéo de

${ }^{6}$ Espace virtuel, Éditions Maison des langues. Consulté le 10 février 2021, disponible sur https://www.emdl.fr/fle/espace-virtuel/ 
phonétique (DF1-CP, 2018). Ces dernières présentent 16 vidéos (2 vidéos pour chacun des 8 dossiers de la méthode) pour faire comprendre et travailler la prononciation qui sont tout aussi bien destinées aux enseignants (au cas où ils n'auraient pas reçu une formation très poussée en la matière ou auraient besoin de guide pour aborder de manière simple et pratique les leçons de phonétique française avec des débutants) qu'aux apprenants (qui arrivent à comprendre les leçons de phonétique grâce aux images, aux animations, aux métaphores et à des comparaisons appropriées). Ces capsules vidéo de phonétique, portant plus sur les éléments suprasegmentaux (surtout sur l'intonation, mais aussi sur l'accentuation, la liaison, l'enchaînement consonantique, l'élision) que segmentaux (sur les voyelles [ə] et [y] et les consonnes [R] et [3]), sans oublier l'aspect morphophonologique du discours (marques orales du singulier/pluriel), ont l'avantage particulier d'être toutes mises en contexte et de toujours faire un lien thématique avec le dossier auquel elles appartiennent. En plus, leur concept novateur combine deux méthodes de correction phonétique ${ }^{7}$ : la méthode articulatoire et la méthode verbo-tonale ${ }^{8}$. Nous prenons ici deux exemples pour illustrer comment on utilise la métaphore d'une maison pour présenter quatre niveaux d'intonation en français :

7 Sur leur choix méthodologique, voir le webinaire «Par quoi commencer en prononciation avec des apprenants débutants? » tenu par Geneviève Briet, une des deux auteures des capsules vidéo de phonétique DF1-CP, organisé en 2019 par Éditions Maison des langues dans le cadre des Rencontres virtuelles FLE. Consulté le 10 février 2021, disponible sur https://www.youtube.com/watch?v=BmyhtY5nfmQ [vidéo]. Voir aussi l'ouvrage La prononciation en classe écrit par les mêmes auteures en collaboration avec Emmanuelle Rassart (Briet-Collige \& Rassart, 2014).

${ }^{8}$ La méthode L'atelier A1 (AT1, 2019), qui s'appuie sur des principes pédagogiques du CECR de 2001 ainsi que sur ses nouveaux descripteurs de 2018, propose aussi 34 capsules vidéo de phonétique (AT1-CP) sur le site de l'éditeur (Didier FLE), mais elles sont essentiellement conçues sur la méthode articulatoire. Consulté le 10 février 2021, disponible sur https://didierfle-latelier.fr/videos-phonetique/ 
Exemple 8a : Activité de phonétique en contexte (DF1-CP, 2018 : Capsule 2)

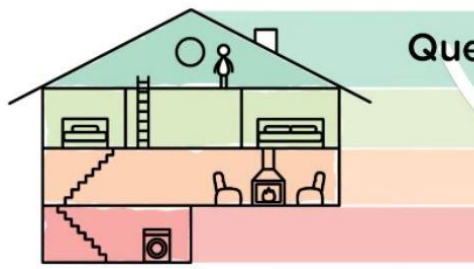

gique?

vous

connaissez- de la Bel

gique ?

Exemple 8b : Activité de phonétique en contexte (DF1-CP, 2018 : Capsule 6)

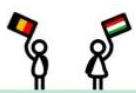

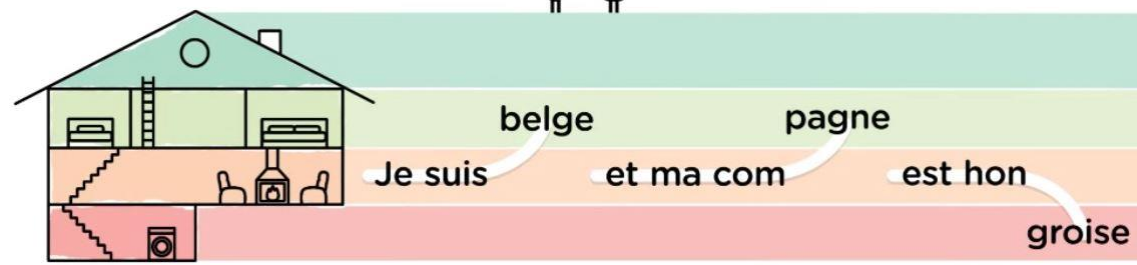

Comme les activités de phonétique, dont un nombre important sont en contexte (64\% hors contexte contre $36 \%$ en contexte), occupent aussi une place importante dans le Cahier d'exercices de la méthode DF1, nous en donnons aussi les deux exemples suivants :

Exemple 9a : Activité de phonétique en contexte (DF1-CA, 2018 : 19)

\section{PROSODIE-Discrimination auditive}

20. Écoutez les phrases suivantes. Pour chaque phrase,

O indiquez sillintonation monte $(-7)$ ou descend ( $)$.

Puis, ajoutez la ponctuation a la fin de chaque phrase.

\begin{tabular}{l|l|l|}
\hline 1. La Zinneke parade, c'est quoi & ( J) (J) \\
\hline 2. C'est un grand défilè multiculturel & \\
\hline 3. Combien ça coùte & \\
\hline 4. C'est gratuit \\
\hline 5. Cela se passe où et quand \\
\hline 6. A Bruxelles, en mai
\end{tabular}

$\uplus_{\text {Lintonation }}$

En génèral, si l'intonation monte (T), c'est une question (?) et si lintonation descend $(\mathrm{D})$, c'est une affirmation $\mathrm{O}$.
Exemple 9b : Activité de phonétique en contexte (DF1-CA, 2018 : 59)

\section{PROSODIE - Intonation et expression}

30. Écoutez et jouez ces dialogues à deux en respectant 7i l'intonationetlexpression.

1. Tu connais cette nouvelle application ?

- Non, pas du tout, montre-moi!

- Regarde, c'est facile et rapide!

2. Jaimerais inviter ma copine au restaurant mais je naai pas assez d’argent.

- Tu peux utiliser fapplication Kidil pour recevoir des offres promotionnelles.

- Trop cool! Merci! 
Résultats statistiques de l'analyse du corpus

Dans notre corpus nous avons relevé au total 370 activités de phonétique (sans compter DF1-CP) dont 191 dans les livres de l'élève et 179 dans les cahiers d'activités. Les données statistiques de notre analyse de leur mise en contexte sont présentées dans le tableau récapitulatif ci-dessous :

Tableau 1 : Résultats statistiques du corpus : des activités de phonétique (en contexte / hors contexte) dans les méthodes de FLE (2011-2018)

\begin{tabular}{|l|c|c|c|c|c|c|}
\hline \multirow{2}{*}{ MÉTHODE } & \multicolumn{3}{|c|}{ Livre de l'élève } & \multicolumn{3}{c|}{ Cahier d'activités } \\
\cline { 2 - 7 } & $\begin{array}{l}\text { Total des } \\
\text { activités }\end{array}$ & $\begin{array}{l}\text { En } \\
\text { contexte }\end{array}$ & $\begin{array}{l}\text { Hors } \\
\text { contexte }\end{array}$ & $\begin{array}{l}\text { Total des } \\
\text { activités }\end{array}$ & $\begin{array}{l}\text { En } \\
\text { contexte }\end{array}$ & $\begin{array}{l}\text { Hors } \\
\text { contexte }\end{array}$ \\
\hline $\begin{array}{l}\text { NRP1, } \\
2011\end{array}$ & 0 & $/$ & $/$ & 25 & $\begin{array}{c}12 \% \\
(3 / 25)\end{array}$ & $\begin{array}{c}88 \% \\
(22 / 25)\end{array}$ \\
\hline COS1, & 81 & $\begin{array}{c}30 \% \\
(24 / 81)\end{array}$ & $\begin{array}{c}70 \% \\
(57 / 81)\end{array}$ & 33 & $\begin{array}{c}9 \% \\
(3 / 33)\end{array}$ & $\begin{array}{c}91 \% \\
(30 / 33)\end{array}$ \\
2017 & 48 & $\begin{array}{c}81 \% \\
(39 / 48)\end{array}$ & $\begin{array}{c}19 \% \\
(9 / 48)\end{array}$ & 20 & $\begin{array}{c}20 \% \\
(4 / 20)\end{array}$ & $\begin{array}{c}80 \% \\
(16 / 20)\end{array}$ \\
\hline ÉC1, & & $\begin{array}{c}48 \% \\
(30 / 62)\end{array}$ & $\begin{array}{c}52 \% \\
(32 / 62)\end{array}$ & 32 & $\begin{array}{c}22 \% \\
(7 / 32)\end{array}$ & $\begin{array}{c}78 \% \\
(25 / 32)\end{array}$ \\
\hline SN1, & 62 & $\begin{array}{c}100 \% \\
(16 / 16)\end{array}$ & $\begin{array}{c}0 \% \\
(0 / 16)\end{array}$ & 69 & $\begin{array}{c}36 \% \\
(25 / 69)\end{array}$ & $\begin{array}{c}64 \% \\
(44 / 69)\end{array}$ \\
\hline DF1, & 169 & & &
\end{tabular}

\section{CONCLUSION}

Les résultats de notre analyse de la mise en contexte des activités de phonétique dans cinq méthodes de FLE (NRP1, COS1, ÉC1, SN1, DF1) nous amènent à tirer au moins trois conclusions. Premièrement, nous constatons que la phonétique reprend de plus en plus d'importance dans les méthodes contemporaines de FLE. Nos résultats montrent que ce n'est que dans les méthodes NRP1 et DF1 que les activités de phonétique ne figurent pas dans le Livre de l'élève, et ce pour des raisons tout à fait différentes. Tandis que dans NRP1 (2011) le rôle de la phonétique semble être marginalisé, dans DF1 (2017) ces activités sont remplacées par des capsules vidéo complètement

9 Il s'agit des capsules de phonétique proposées dans le Livre de l'élève du DF1, disponibles gratuitement sur le site de l'Espace virtuel des Éditions Maison des langues (DF1-CP, 2018). Consulté le 10 février 2021, disponible sur https://www.emdl.fr/fle/espace-virtuel/. 
mises en contexte et en plus, cette dernière méthode se distingue de toutes les autres par un plus grand nombre d'activités de phonétique dans le Cahier d'exercices dont plus d'un tiers est mis en contexte. Deuxièmement, nous remarquons un effort considérable chez la majorité des auteurs pour contextualiser les activités de phonétique. Les auteurs se servent de plusieurs modalités pour y réussir : entre autres choses, en incorporant les activités de phonétique dans d'autres activités de compréhension et expression orales du livre de l'élève (SN1). Comme les cahiers d'activités ont en général l'objectif de " renforcer la systématisation » et de " consolider les compétences linguistiques » (NRP1-CA, 2011: 3) à travers "l'automatisation » des structures linguistiques (ÉC1-GP, 2013 : V), ils proposent des activités que sont moins contextualisées que celles des livres de l'élève. Finalement, nous constatons que les auteurs des méthodes de FLE s'efforcent de rendre les activités de phonétique intéressantes aux apprenants. Afin d'éviter la monotonie des exercices répétitifs et mécaniques, de rendre les apprenants plus actifs et de les aider à découvrir et à comprendre les phénomènes phonétiques, ils proposent des documents captifs (de courtes vidéos avec une riche animation), des activités ludiques, des activités de production orale, etc. Tout ceci nous encourage à dire que nous sommes désormais loin de l'époque où la phonétique était considérée comme " un vilain petit canard de la didactique » (Billières, 2014, para. 1) et que les activités de phonétique dans les méthodes contemporaines de FLE sont de plus en plus élaborées et contextualisées.

Nataša Radusin Bardić

ON THE CONTEXTUALIZATION OF PHONETIC EXERCISES IN COURSES OF FRENCH AS A FOREIGN LANGUAGE BASED ON THE ACTION-ORIENTED APPROACH

\section{Summary}

Our research includes an analysis of the contextualization of phonetic exercises in five courses of French as a foreign language which were published between 2011 and 2018 following the principles of the communicative and action-oriented approaches and relying on the guidelines of the Common European Framework of Reference for Languages (CEFR) of 2001 (Nouveau Rond-Point 1, Écho A1, Saison 1, Cosmopolite 1 and Défi 1). The goal of our research is twofold. On the one hand, we would like to find out what importance is given to phonetic exercises in each of the mentioned courses. On the other hand, our goal is to establish the extent to which phonetic exercises are used 
in the context, bearing in mind the fact that it is context that gets great attention within CEFR, in every form of use and learning a foreign language. For the purposes of facilitating the comparison of the obtained results and better understanding of the contextualization of the phonetic exercises in the selected courses, our analysis is based on both qualitative and quantitative criteria. A total of 370 phonetic exercises were recorded in our corpus, 191 of which were in the analyzed textbooks and 179 being in the complementary workbooks. Based on the obtained results, we can draw at least three conclusions. First of all, we can notice that phonetics is gaining more and more importance in modern courses of French as a foreign language. Our results show that only the courses Nouveau Rond-Point 1 and Défi 1 do not feature phonetic exercises in their textbooks, but this comes from completely different reasons. While in Nouveau Rond-Point 1 (2011) the role of phonetics seems to be marginalized, in Défi 1 (2017) the lack of these exercises in the textbook is compensated by the abundant additional material: the short videos available on the publisher's website in which the phonetic material is explained in a contextualized way (through animations, metaphors, comparisons) and it is quite thematically harmonized with the lesson referred to. In addition, the workbook of the course Défi 1 was noted to feature the largest number of phonetic exercises of all the analyzed courses, and a significant part of those exercises was also contextualized. As for our next conclusion, the fact is imposed that there is a noticeable tendency towards contextualization of phonetic exercises in modern courses of French as a foreign language. The authors of the courses achieve this in various ways - among other things, by inserting phonetic exercises into other exercises of oral comprehension or expression, as is the case with the course Saison 1. Finally, we can conclude that in modern courses of French as a foreign language more attention is paid to how to motivate students to do as many phonetic exercises as possible. In order to avoid the monotony of mechanical repetition exercises, and in order for students to be more active and better understand phonetic phenomena, there are additional audio-visual materials, learning through play, phonetic exercises within oral comprehension and expression exercises available to them. All this encourages us to say that phonetics today is far from being marginalized, as was the case at the very beginning of the introduction of a communicative approach in learning/teaching French as a foreign language.

Key words: French as a foreign language, phonetics of the French language, phonetic exercises, contextualization, communicative approach, action-oriented approach.

\section{RÉFÉRENCES BIBLIOGRAPHIQUES}

Abry, D.-Veldeman-Abry, J. (2007). La phonétique. Audition, prononciation, correction. Paris : CLE International. 
Bertocchini, P.-Costanzo, E. (2008). Manuel de formation pratique pour le professeur de FLE. Paris : CLE International.

Briet, G. (2019). Par quoi commencer en prononciation avec des apprenants débutants ? [vidéo]. Rencontres virtuelles FLE. Éditions Maison des langues. Consulté le 10 février 2021, disponible sur https://www.youtube.com/watch?v=BmyhtY5nfmQ

Briet, G.-Collige, V. \& Rassart, E. (2014). La prononciation en classe. Grenoble : Presses universitaires de Grenoble, FLE.

Billières, M. (2014). La phonétique, vilain petit canard de la didactique. Au son du FLE [blog]. Consulté le 10 février 2021, disponible sur https://www.verbotonale-phonetique.com/phonetique-didactique/

Callamand, M.-Pedoya, E. (1984). Phonétique et enseignement. Le Français dans le monde, 182, 56-58.

Champagne-Muzar, C.-Bourdages, J. S. (1998). Le point sur la phonétique. Paris : CLE International.

CECR : Conseil de l'Europe. (2001). Cadre européen commun de référence pour les langues : apprendre, enseigner, évaluer (CECR). Strasbourg : Unité de Politiques Linguistiques. Consulté le 3 février 2021, disponible sur https://rm.coe.int/16802fc3a8

Conseil de l'Europe. (2018). Cadre européen commun de référence pour les langues : apprendre, enseigner, évaluer. Volume complémentaire avec de nouveaux descripteurs. Strasbourg : Programme des Politiques Linguistiques, Division des Politiques éducatives, Service de l'Éducation. Consulté le 3 février 2021, disponible sur https://rm.coe.int/cecr-volume-complementaire-avec-de-nouveauxdescripteurs/16807875d5

Cuq, J.-P. (dir.). (2003). Dictionnaire de didactique du français langue étrangère et seconde. Paris : CLE International.

Cuq, J.-P.-Gruca, I. (2008). Cours de didactique du français langue étrangère et seconde. Grenoble : Presses universitaires de Grenoble.

Galazzi-Matasci, E.-Pedoya, E. (1983). Et la pédagogie de la prononciation ? Le Français dans le monde, 180, 39-44.

Guimbretière, É. (1994). Phonétique et enseignement de l'oral. Paris : Didier/Hatier.

Lauret, B. (2007). Enseigner la prononciation du français : questions et outils. Paris : Hachette FLE. 
Porcher, L. (1987). Simples propos d'un usager. Études de linguistique appliquée, 66, 134-145.

Robert, J.-P. (2008). Dictionnaire pratique de didactique du FLE. Paris : Ophrys.

Robert, J.-P.-Rosen, É. \& Reinhardt, C. (2011). Faire classe en FLE. Une approche actionnelle et pragmatique. Paris : Hachette.

Trim, J. (dir.). (2001). Cadre européen commun de référence pour les langues : apprendre, enseigner, évaluer. Guide pour les utilisateurs. Strasbourg : Division des Politiques Linguistiques. Consulté le 17 juillet 2021, disponible sur https://rm.coe.int/168069782b

Corpus : méthodes de FLE

AT1 : Cocton, M.-N.-Pommier, É.-Ripaud, D. \& Rabin, M. (2019). L'atelier. A1. Méthode de français. [Livre de l'élève]. Paris : Didier.

AT1-CP : Ripaud, D. (2018). L'atelier A1. Vidéos de phonétique. Éditions Didier FLE. [Wacky, W. - réalisation et montage, Beausivoir, G - soustitrage pour EuroDVD]. Consulté le 10 février 2021, disponible sur https://didierfle-latelier.fr/videos-phonetique/

AT1-GP : Cocton, M.-N.-Pommier, É. (2019). L'atelier. A1. Méthode de français. Guide pratique de classe. Paris : Didier.

COS1 : Hirschsprung, N.-Tricot, T. (2017). Cosmopolite. A1. Méthode de français. [Livre de l'élève]. Paris : Hachette FLE.

COS1-CA : Hirschsprung, N.-Mater, A.-Mathieu-Benoit, É.-Mous, N. \& Tricot, T. (2017). Cosmopolite. A1. Méthode de français. Cahier d'activités. Paris : Hachette FLE.

COS1-GP : Antier, M.-Garcia, E.-Gaudel, A.-Mous, N. \& Veillon-Leroux, A. (2017). Cosmopolite. A1. Méthode de français. Guide pédagogique. Paris : Hachette FLE.

DF1 : Chahi, F.-Denyer, M.-Gloanec, A.-Briet, G.-Collige-Neuenschwander, V. \& Fouillet, R. (2018). Défi 1. A1. Méthode de français. [Livre de l'élève]. Paris : Éditions Maison des langues.

DF1-CA: Chahi, F.-Denyer, M.-Gloanec, A.-Rongé, C. de-Verhulst, N. \& Horquin, A. (2018). Défi 1. A1. Méthode de français. Cahier d'exercices. Paris : Éditions Maison des langues.

DF1-CP : Briet, G.-Collige-Neuenschwander, V. (2018). Défi 1. Ressources : Capsules de phonétique [vidéo]. Espace virtuel: Nos manuels et 
ressources. Éditions Maison des langues. Consulté le 10 février 2021, disponible sur https://www.emdl.fr/fle/espace-virtuel/

DF1-GP : Denyer, M. (2018). Défi 1. A1. Méthode de français. Guide pédagogique.

Paris : Éditions Maison des langues.

ÉC1 : Girardet, J.-Pécheur, J. (2013). Écho A1. Méthode de français. [Livre de l'élève, $2^{\mathrm{e}}$ édition.] Paris : CLE International.

ÉC1-CA : Girardet, J.-Pécheur, J. (2013). Écho A1. Méthode de français. Cahier personnel d'apprentissage. Paris : CLE International.

ÉC1-GP : Girardet, J.-Pécheur, J. (2013). Écho A1. Méthode de français. Livre du professeur. [2e édition.]. Paris : CLE International.

ÉD1 : Alcaraz, M.-Braud, C.-Calvez, A.-Cornuau, G.-Jacob, A.-Pinson, C. \& Vidal, S. (2016). Édito. Niveau A1. Méthode de français. [Livre de l'élève]. Paris : Didier.

ÉD1-GP : Braud, C. (2016). Édito. Niveau A1. Guide pédagogique. Paris : Didier. NRP1 : Flumian, C.-Labascoule, J.-Lause, C. \& Royer, C. (2011). Nouveau RondPoint 1. A1-A2. Méthode de français basée sur l'apprentissage par les tâches. [Livre de l'élève]. Paris : Éditions Maison des langues.

NRP1-CA : Flumian, C.-Labascoule, J.-Liria, P.-Rodriguez, M. R. \& Royer, C. (2011). Nouveau Rond-Point 1. A1-A2. Méthode de français basée sur l'apprentissage par les tâches. Cahier d'activités + CD audio. Paris : Éditions Maison des langues.

NRP1-GP : Denyer, M.-Lhuilier, N. (2011). Nouveau Rond-Point 1. Guide pédagogique. Paris : Éditions Maison des langues.

SN1 : Cocton, M.-N.-Heu, É.-Houssa, C.-Kasazian, É.-Dupleix, D. \& Ripaud, D. (2014). Saison 1. A1+. Méthode de français. [Livre de l'élève]. Paris : Didier.

SN1-CA : Alcaraz, M.-Escoufier, D.-Gomy, C.-Landier, M.-Quéméner, F. \& Ripaud, D. (2014). Saison 1. A1+. Méthode de français. Cahier d'activités. Paris : Didier.

SN1-GP : Cocton, M.-N.-Pommier, É. (2014). Saison 1. A1+. Méthode de français. Guide pédagogique. Paris : Didier. 